\title{
Sex-dependent vulnerability to Cannabis abuse in adolescence
}

\author{
Tiziana Rubino* and Daniela Parolaro \\ Department of Theoretical and Applied Sciences, and Neuroscience Center, University of Insubria, Busto Arsizio, Italy
}

The goal of this review is to summarize current evidence for sex differences in the response to cannabinoid compounds, focusing mainly on a specific age of exposure, i.e., adolescence. Preclinical as well as clinical studies are examined. Among the different possible underlying mechanisms, the consistent dimorphism in the endocannabinoid system and delta9-tetrahydrocannabinol metabolism may play a part. All the collected data point to the need of including females in basic research as well as of analyzing results for sex differences in epidemiological studies.

Keywords: Cannabis abuse, adolescence, sex, human studies, animal models

\section{OPEN ACCESS}

Edited by:

Liana Fattore,

National Research Council, Italy

Reviewed by:

Caroline Davis,

York University, Canada

Fernando Rodriguez de Fonseca, Instituto de Investigación Biomédica de Málaga, Spain

*Correspondence: Tiziana Rubino

Department of Theoretical and Applied Sciences, and Neuroscience

Center, University of Insubria, Via Manara 7, Busto Arsizio 21052, Italy tiziana.rubino@uninsubria.it

Specialty section:

This article was submitted to Addictive Disorders and Behavioral Dyscontrol, a section of the journal

Frontiers in Psychiatry

Received: 03 February 2015

Accepted: 31 March 2015

Published: 20 April 2015

Citation:

Rubino T and Parolaro D (2015)

Sex-dependent vulnerability to

Cannabis abuse in adolescence.

Front. Psychiatry 6:56.

doi: 10.3389/fpsyt.2015.00056

\section{Introduction}

Cannabis continues to be the most widely used illicit substance among adolescents in the world, and more users are seeking treatment each year (1). Accumulating evidence suggests that exposure to Cannabis or its psychoactive ingredient delta9-tetrahydrocannabinol (THC) during the adolescent developmental window may act as a risk factor for the occurrence of psychiatric disorders later in life (2-4).

Despite the well-accepted notion that several neuropsychiatric disorders, such as depression, conduct problems, and autism, are sex-related [see, for review, Ref. (5-7)], very few papers have dealt with sex vulnerability to adolescent Cannabis abuse, both at the preclinical and clinical level. The main obstacle to this lies in the fact that research is still mainly focused on the male sex: male animals in preclinical research and male subjects in clinical studies. The potential sex influence is still routinely ignored or dismissed even when both sexes are included, as in some human studies where no sex-related analysis is performed, but all the subjects are regarded as "unisex." Fortunately, the view that biological sex is unimportant in neuroscience is increasingly seen as a false assumption [see for a commentary Ref. (8)]. Notably, the National Institute of Health has recently asked the scientific community for sex and gender inclusion plans in preclinical research (9).

We hope from now on to witness an increasing amount of research considering both sexes. However, so far, few papers have dealt with the influence of this variable on the response to cannabinoids during adolescence. Most work has been done at the preclinical level, but some literature on humans is now also appearing. For the sake of accuracy, in this review we will take into account only papers where both male and females are considered, or papers applying exactly the same paradigm of exposure in male and female animals.

\section{Human Studies}

Few studies exist on sex-dependent effects of adolescent Cannabis abuse in humans, so it is difficult to draw a precise picture of this phenomenon. Nonetheless, here we want to discuss some interesting observations. Generally, Cannabis use is more prevalent among males, who display an earlier age of onset of use and are more likely to be on a heavier use trajectory (10). 
As a consequence, males appear to be more likely than females to become dependent on Cannabis $(11,12)$. However, females tend to have shorter intervals between the onset of use and regular use or development of dependence $(13,14)$. Accordingly, females enter treatment for Cannabis use disorders after fewer years and less cumulative use compared to males (15). In general, Cannabis abuse is associated with a broad range of adverse health measures in both adolescent girls and boys (14). The existence of an overall sex-dependent effect has already been reported for other drugs of abuse (16), and specifically, female adolescent users seem to experience negative consequences of drug use earlier than male peers, and appear to be more likely to suffer from an internalizing disorder, such as depressive and anxiety disorders (16). Conversely, male substance abusers have more externalizing behaviors, such as aggressiveness and impulsivity (16). This seems to be true also for Cannabis. One of the first papers describing this correlation reported that daily Cannabis use was associated with a fivefold increase in anxiety and depression in young females, but not males (17). Accordingly, higher rates of comorbid mood and anxiety disorders in women have been recently observed in a large epidemiological study performed in the United States (18). Adolescent female abusers, who developed greater internalizing symptoms, exhibited larger right amygdala volumes relative to males and female controls (19). Interestingly, larger amygdala volumes were associated with increased depression and anxiety symptomatology (19). Similarly, Lai and Sitharthan (20) reported a significant association between Cannabis use disorder and mental health disorders, and again, higher comorbidity rates were observed for females. The most common mental disorders were major depression, personality disorder, schizophrenia, and severe stress disorder (20). Potential sex-differences have also been reported for Cannabis use and neurocognitive functioning (21). Specifically, Cannabis use was more consistently associated with poorer episodic memory performance in females and with poorer decision-making performance in males. Female Cannabis users presented a larger prefrontal cortex (PFC) volume compared to controls, whereas male users presented a smaller one (22). It is worth noting that among users, larger PFC total volume was associated with worse executive functioning, thus implying that females performed the worst. Finally, studying the association between Cannabis use and earlier age of onset of psychosis (AOP), researchers found that male users are the group with the earliest AOP. However, this seems to be independent of sex, and instead linked to the fact that males start first and consume more than females (23).

In conclusion, Cannabis abuse in humans appears to be associated with different responses in male and females, resembling what has already been seen with other drugs of abuse. The molecular bases of these sex differences need further investigation. Future studies should take into account the interaction between the endocannabinoid system and sex hormones, but also the fact that adolescent males and females undergo neuromaturation at separate rates, thus presenting differential trajectories of neuronal maturation at the same age $(24,25)$, that could hence be differently affected by Cannabis.

\section{Animal Studies}

Animal models, although far from addressing the complexity of human disorders, allow experimental controls that are not possible in human studies. Moreover, they provide a valuable approach for the investigation of neurobiological substrates. Through this helpful tool, it has been confirmed that chronic administration of natural or synthetic cannabinoids during the adolescent period - using paradigms resembling heavy Cannabis abuse in humans - causes persistent behavioral alterations in adult animals [see, for review, Ref. $(2,4,26)]$. Cognition is one of the most explored brain functions after adolescent exposure to natural or synthetic cannabinoids. When sex was taken into account, it appeared that cannabinoid exposure during adolescence impaired learning and memory in both sexes. O'Shea et al. $(27,28)$ demonstrated that adolescent exposure to increasing doses of the synthetic cannabinoid agonist CP-55,940 for 21 days induced impaired recognition memory in the novel object recognition test long after discontinuation of the drug, in both female and male rats. However, when spatial memory was assessed in the Morris water maze test, adolescent cannabinoid exposure in both sexes disrupted learning immediately after the treatment (29), but not after a long drug-free period $(29,30)$. In the active place avoidance (APA) paradigm, where animal's ability to learn and retrieve spatial information as well as flexibility of learning is assessed, early adolescent THC exposure did not affect the task acquisition, nor the performance after the 24-h retention interval in adult animals of both sexes (31). However, when flexibility was considered, impaired performance on the reversal trial of the APA task was observed (31). In the radial maze test, used to assess spatial working memory, both male and female rats showed deficits when tested long after adolescent exposure to $\operatorname{THC}(32,33)$. These data suggest that adolescent exposure to cannabinoids induces longterm cognitive impairments specifically in recognition and spatial working memory, as well as in flexibility, whereas pure spatial memory does not seem to be affected. However, these effects do not display sex differences, since they are present in both male and female animals. Less consistent results have been obtained about the impact of adolescent cannabinoid treatment on anxiety behaviors. In fact, results coming from adult animals of both sexes exposed to cannabinoids during their adolescence showed all type of responses: anxiolytic-like response (34), anxiogenic-like effect $(27,28)$, or no changes in their behavior $(35)$. Neither conclusions regarding the impact of adolescent exposure on anxiety behaviors nor about possible sex differences can be drawn from these findings. A different picture is present when the forced swim test was used: adolescent exposure to THC induced a significant increase in immobility that was apparent only in female rats $(35,36)$. Also, the effect of adolescent cannabinoid exposure on adult drug selfadministration seems to present sex-dependency. Higher adult cocaine self-administration rates have been reported in female rats only (37), whereas increase in morphine self-administration under the fixed ratio 1 schedule has been described in males but not in females (38). As a whole, animal models seem to confirm the existence of some sex-dependent responses to adolescent cannabinoid exposure, with females appearing more sensitive than males in the emotional sphere. 
These differences in behavior are substantiated by differences at the cellular/molecular level. Pharmacokinetics seems to play a part. It has been recently reported that adolescent female rats exhibit pronounced metabolism of THC to the still active compound 11-OH-THC compared to their male conspecifics, particularly after repeated THC administration (39). Thus, THC exposure could conceivably be potentiated by its active metabolite in female adolescents. This fact together with the observation that adolescent female rats possess more efficient $\mathrm{CB} 1$ receptors (40), suggests that they may be more vulnerable to THC effects. Accordingly, chronic THC exposure in adolescence induced more intense $\mathrm{CB} 1$ receptor desensitization in females, with more brain areas involved, despite similar down-regulation $(35,41)$. If confirmed also in humans, this would explain, at least in part, why females tend to have shorter intervals between the onset of use and the development of dependence, the so-called "telescoping effect" $(13-15,18)$. Another observation that comes from animal studies and deserves further investigation is that sex-dependent sensitivity appears to exist also with regard to the brain regions that are affected by the treatment. Specifically, in female animals, among all the cerebral areas investigated, the PFC seems to be the most affected, whereas it is the hippocampus in males. For example, Higuera-Matas et al. (30) reported that while periadolescent exposure to a fixed dose of a synthetic cannabinoid agonist did not produce robust behavioral effects, it did induce an increase of the plasticity marker PSA-NCAM in the hippocampus of males only. Similarly, Lee et al. (42) showed that a sustained adolescent $\mathrm{CB} 1$ receptor activation reduced adult hippocampal neurogenesis in both sexes; however, for some parameters, males appeared to be more greatly affected than females. Our group, in the search for a possible molecular correlate for the impaired spatial working memory induced by adolescent THC administration, investigated some markers of neuroplasticity in the PFC and hippocampus of both male and female rats (32, 33). Interestingly, a significant decrease in pre- and post-synaptic markers was present in the hippocampus of male rats, whereas the same proteins changed in the PFC of female animals (32, 33). Of note, in human Cannabis abusers, the occurrence of significant changes in the hippocampus of males (43) and in the PFC and amygdala of females $(19,22)$ have been observed. These brain regions are differently involved in the modulation of cognition (hippocampus and PFC) and emotion (amygdala and PFC), and this may explain the greater effect on emotionality in females.

\section{References}

1. United Nations Office on Drugs and Crime (UNODC). World Drug Report 2014. New York, NY: United Nations (2014).

2. Rubino T, Zamberletti E, Parolaro D. Adolescent exposure to cannabis as a risk factor for psychiatric disorders. J Psychopharmacol (2012) 26:177-88. doi:10. $1177 / 0269881111405362$

3. Chadwick B, Miller ML, Hurd YL. Cannabis use during adolescent development: susceptibility to psychiatric illness. Front Psychiatry (2013) 4:129. doi:10. 3389/fpsyt.2013.00129

4. Renard J, Krebs MO, Le Pen G, Jay TM. Long-term consequences of adolescent cannabinoid exposure in adult psychopathology. Front Neurosci (2014) 8:361. doi:10.3389/fnins.2014.00361

\section{Conclusion}

In conclusion, some sex-dependent effects exist in the response to cannabinoid compounds between adolescent males and females. These effects may rely on the different pharmacokinetics described for THC between males and females as well as on sex differences present in the endocannabinoid system. To complicate the picture, a fact that is specific for the adolescent population and should also be taken into account is represented by the observation that some brain developmental characteristics are different in the two sexes. For example, neurodevelopmental trajectories are significantly different between males and females [(25); see, for review, Ref. (44)]. Total brain size and regional gray matter volumes follow an inverted $U$ shaped maturational curve and peak earlier in females, thus suggesting that the pruning process occurring in the adolescent brain might be present with different intensity in boys and girls of the same age. Since it has been recently suggested that the endocannabinoid system in the adolescent brain may play a part in synaptic pruning (45), exposure to cannabinoids during adolescence might differently interact with the pruning event in boys and girls, thus leading to different impairments in brain and behavior. Not least, interactions of the endocannabinoid system with gonadal hormones may also play a part. Interestingly, it has been recently suggested that sex hormones and the endocannabinoid system might work in symphony to promote maturational processes within the adolescent brain, specifically in those circuits important for the emotional and motivational response to sexually relevant stimuli (46). However, the existence of a close interaction between the endocannabinoid system and sex hormones has long been known. For example, CB1 receptor expression and density appear to be under the control of sex steroids in both males and females in some cerebral areas (47, 48). More recently, it has been reported that endocannabinoids and gonadal hormones may reciprocally regulate each other, and interestingly, estrogen can recruit endocannabinoids to modulate emotionality $(49,50)$. This is particularly important when considering that ovarian hormones may actively contribute to the remodeling event in the female brain during puberty and adolescence, as recently suggested by Juraska et al. (51). This was demonstrated for few brain areas; among them, there are the PFC and amygdala, the very same areas mainly affected by cannabinoids in adolescent females. A deeper knowledge of all these interactions would be helpful in designing proper sexspecific treatments or interventions to prevent or recover the long-term adverse effects induced by adolescent heavy Cannabis abuse.

5. Andersen SL. Trajectories of brain development: point of vulnerability or window of opportunity? Neurosci Biobehav Rev (2003) 27:3-18. doi:10.1016/ S0149-7634(03)00005-8

6. Zahn-Waxler C, Shirtcliff EA, Marceau K. Disorders of childhood and adolescence: gender and psychopathology. Annu Rev Clin Psychol (2008) 4:275-303. doi:10.1146/annurev.clinpsy.3.022806.091358

7. Solomon MB, Herman JP. Sex differences in psychopathology: of gonads, adrenals and mental illness. Physiol Behav (2009) 97:250-8. doi:10.1016/j. physbeh.2009.02.033

8. Cahill L. Fundamental sex difference in human brain architecture. Proc Nat Acad Sci U S A (2014) 111:577-8. doi:10.1073/pnas.1320954111

9. Clayton JA, Collins FS. Policy: NIH to balance sex in cell and animal studies. Nature (2014) 509:282-3. doi:10.1038/509282a 
10. Kandel DB, Chen K. Types of marijuana users by longitudinal course. J Stud Alcohol (2000) 61:367-78. doi:10.15288/jsa.2000.61.367

11. Wagner FA, Anthony JC. Male-female differences in the risk of progression from first use to dependence upon cannabis, cocaine, and alcohol. Drug Alcohol Depend (2007) 86:191-8. doi:10.1016/j.drugalcdep.2006.06.003

12. Farmer RF, Kosty DB, Seeley JR, Duncan SC, Lynskey MT, Rohde P, et al. Natural course of cannabis use disorders. Psychol Med (2015) 45:63-72. doi:10. 1017/S003329171400107X

13. Ridenour TA, Lanza ST, Donny EC, Clark DB. Different lengths of times for progressions in adolescent substance involvement. Addict Behav (2006) 31:962-83. doi:10.1016/j.addbeh.2006.03.015

14. Schepis TS, Desai RA, Cavallo DA, Smith AE, McFetridge A, Liss TB, et al. Gender differences in adolescent marijuana use and associated psychosocial characteristics. J Addict Med (2011) 5:65-73. doi:10.1097/ADM. 0b013e3181d8dc62

15. Hernandez-Avila CA, Rounsaville BJ, Kranzler HR. Opioid-, cannabis- and alcohol-dependent women show more rapid progression to substance abuse treatment. Drug Alcohol Depend (2004) 74:265-72. doi:10.1016/j.drugalcdep. 2004.02.001

16. Kloos A, Weller RA, Chan R, Weller EB. Gender differences in adolescent substance abuse. Curr Psychiatry Rep (2009) 11:120-6. doi:10.1007/ s11920-009-0019-8

17. Patton GC, Coffey C, Carlin JB, Degenhardt L, Lynskey M, Hall W. Cannabis use and mental health in young people: cohort study. BMJ (2002) 325:1195-8. doi:10.1136/bmj.325.7374.1195

18. Khan SS, Secades-Villa R, Okuda M, Wang S, Pérez-Fuentes G, Kerridge BT, et al. Gender differences in cannabis use disorders: results from the National Epidemiologic Survey of Alcohol and Related Conditions. Drug Alcohol Depend (2013) 130:101-8. doi:10.1016/j.drugalcdep.2012.10.015

19. McQueeny T, Padula CB, Price J, Medina KL, Logan P, Tapert SF. Gender effects on amygdala morphometry in adolescent marijuana users. Behav Brain Res (2011) 224:128-34. doi:10.1016/j.bbr.2011.05.031

20. Lai HM, Sitharthan T. Exploration of the comorbidity of cannabis use disorders and mental health disorders among inpatients presenting to all hospitals in New South Wales, Australia. Am J Drug Alcohol Abuse (2012) 38:567-74. doi:10. 3109/00952990.2012.694523

21. Crane NA, Schuster RM, Gonzalez R. Preliminary evidence for a sex-specific relationship between amount of cannabis use and neurocognitive performance in young adult cannabis users. J Int Neuropsychol Soc (2013) 19:1009-15. doi:10. 1017/S135561771300088X

22. Medina KL, McQueeny T, Nagel BJ, Hanson KL, Yang TT, Tapert SF. Prefrontal cortex morphometry in abstinent adolescent marijuana users: subtle gender effects. Addict Biol (2009) 14:457-68. doi:10.1111/j.1369-1600.2009.00166.x

23. Di Forti M, Sallis H, Allegri F, Trotta A, Ferraro L, Stilo SA, et al. Daily use, especially of high-potency cannabis, drives the earlier onset of psychosis in cannabis users. Schizophr Bull (2014) 40:1509-17. doi:10.1093/schbul/sbt181

24. Nagel BJ, Medina KL, Yoshii J, Schweinsburg AD, Moadab I, Tapert SF. Age-related changes in prefrontal white matter volume across adolescence. Neuroreport (2006) 17:1427-31. doi:10.1097/01.wnr.0000233099.97784.45

25. Lenroot RK, Gogtay N, Greenstein DK, Wells EM, Wallace GL, Clasen LS, et al. Sexual dimorphism of brain developmental trajectories during childhood and adolescence. Neuroimage (2007) 36:1065-73. doi:10.1016/j.neuroimage.2007. 03.053

26. Realini N, Rubino T, Parolaro D. Neurobiological alterations at adult age triggered by adolescent exposure to cannabinoids. Pharmacol Res (2009) 60:132-8. doi:10.1016/j.phrs.2009.03.006

27. O'Shea M, Singh ME, McGregor IS, Mallet PE. Chronic cannabinoid exposure produces lasting memory impairment and increased anxiety in adolescent but not adult rats. J Psychopharmacol (2004) 18:502-8. doi:10.1177/ 0269881104047277

28. O'Shea M, McGregor IS, Mallet PE. Repeated cannabinoid exposure during perinatal, adolescent or early adult ages produces similar longlasting deficits in object recognition and reduced social interaction in rats. J Psychopharmacol (2006) 20:611-21. doi:10.1177/0269881106065188

29. Cha YM, Jones KH, Kuhn CM, Wilson WA, Swartzwelder HS. Sex differences in the effects of delta9-tetrahydrocannabinol on spatial learning in adolescent and adult rats. Behav Pharmacol (2007) 18:563-9. doi:10.1097/FBP. 0b013e3282ee7b7e
30. Higuera-Matas A, Botreau F, Miguéns M, Del Olmo N, Borcel E, PérezAlvarez L, et al. Chronic periadolescent cannabinoid treatment enhances adult hippocampal PSA-NCAM expression in male Wistar rats but only has marginal effects on anxiety, learning and memory. Pharmacol Biochem Behav (2009) 93:482-90. doi:10.1016/j.pbb.2009.06.013

31. Harte LC, Dow-Edwards D. Sexually dimorphic alterations in locomotion and reversal learning after adolescent tetrahydrocannabinol exposure in the rat. Neurotoxicol Teratol (2010) 32:515-24. doi:10.1016/j.ntt.2010.05.001

32. Rubino T, Realini N, Braida D, Alberio T, Capurro V, Viganò D, et al. The depressive phenotype induced in adult female rats by adolescent exposure to THC is associated with cognitive impairment and altered neuroplasticity in the prefrontal cortex. Neurotox Res (2009) 15:291-302. doi:10.1007/ s12640-009-9031-3

33. Rubino T, Realini N, Braida D, Guidi S, Capurro V, Viganò D, et al. Changes in hippocampal morphology and neuroplasticity induced by adolescent THC treatment are associated with cognitive impairment in adulthood. Hippocampus (2009) 19:763-72. doi:10.1002/hipo.20554

34. Biscaia M, Marín S, Fernández B, Marco EM, Rubio $M$, Guaza $C$, et al. Chronic treatment with CP 55,940 during the peri-adolescent period differentially affects the behavioural responses of male and female rats in adulthood. Psychopharmacology (2003) 170:301-8. doi:10.1007/s00213-003-1550-7

35. Rubino T, Vigano' D, Realini N, Guidali C, Braida D, Capurro V, et al. Chronic delta 9-tetrahydrocannabinol during adolescence provokes sex-dependent changes in the emotional profile in adult rats: behavioral and biochemical correlates. Neuropsychopharmacology (2008) 33:2760-71. doi:10.1038/sj.npp. 1301664

36. Zamberletti E, Prini P, Speziali S, Gabaglio M, Solinas M, Parolaro D, et al. Gender-dependent behavioral and biochemical effects of adolescent delta-9tetrahydrocannabinol in adult maternally deprived rats. Neuroscience (2012) 204:245-57. doi:10.1016/j.neuroscience.2011.11.038

37. Higuera-Matas A, Soto-Montenegro ML, del Olmo N, Miguéns M, Torres I, Vaquero JJ, et al. Augmented acquisition of cocaine self-administration and altered brain glucose metabolism in adult female but not male rats exposed to a cannabinoid agonist during adolescence. Neuropsychopharmacology (2008) 33:806-13. doi:10.1038/sj.npp.1301467

38. Biscaia M, Fernández B, Higuera-Matas A, Miguéns M, Viveros MP, GarcíaLecumberri $C$, et al. Sex-dependent effects of periadolescent exposure to the cannabinoid agonist CP-55,940 on morphine self-administration behaviour and the endogenous opioid system. Neuropharmacology (2008) 54:863-73. doi:10. 1016/j.neuropharm.2008.01.006

39. Wiley JL, Burston JJ. Sex differences in $\Delta(9)$-tetrahydrocannabinol metabolism and in vivo pharmacology following acute and repeated dosing in adolescent rats. Neurosci Lett (2014) 576:51-5. doi:10.1016/j.neulet.2014.05. 057

40. Rubino T, Parolaro D. Sexually dimorphic effects of cannabinoid compounds on emotion and cognition. Front Behav Neurosci (2011) 28(5):64. doi:10.3389/ fnbeh.2011.00064

41. Burston JJ, Wiley JL, Craig AA, Selley DE, Sim-Selley LJ. Regional enhancement of cannabinoid CB1 receptor desensitization in female adolescent rats following repeated Delta-tetrahydrocannabinol exposure. Br J Pharmacol (2010) 161:103-12. doi:10.1111/j.1476-5381.2010.00870.x

42. Lee TT, Hill MN, Hillard CJ, Gorzalka BB. Temporal changes in Nacylethanolamine content and metabolism throughout the peri-adolescent period. Synapse (2013) 67:4-10. doi:10.1002/syn.21609

43. Solowij N, Walterfang M, Lubman DI, Whittle S, Lorenzetti V, Styner M, et al. Alteration to hippocampal shape in cannabis users with and without schizophrenia. Schizophr Res (2013) 143:179-84. doi:10.1016/j.schres.2012. 10.040

44. Lenroot RK, Giedd JN. Sex differences in the adolescent brain. Brain Cogn (2010) 72:46-55. doi:10.1016/j.bandc.2009.10.008

45. Rubino T, Prini P, Piscitelli F, Zamberletti E, Trusel M, Melis M, et al. Adolescent exposure to THC in female rats disrupts developmental changes in the prefrontal cortex. Neurobiol Dis (2015) 73:60-9. doi:10.1016/j.nbd.2014. 09.015

46. Chadwick B, Saylor AJ, López HH. Adolescent cannabinoid exposure attenuates adult female sexual motivation but does not alter adulthood CB1R expression or estrous cyclicity. Pharmacol Biochem Behav (2011) 100:157-64. doi:10.1016/ j.pbb.2011.07.006 
47. Rodríguez de Fonseca F, Cebeira M, Ramos JA, Martín M, Fernández-Ruiz JJ. Cannabinoid receptors in rat brain areas: sexual differences, fluctuations during estrous cycle and changes after gonadectomy and sex steroid replacement. Life Sci (1994) 54:159-70. doi:10.1016/0024-3205(94)00585-0

48. González S, Bisogno T, Wenger T, Manzanares J, Milone A, Berrendero F, et al. Sex steroid influence on cannabinoid $\mathrm{CB}(1)$ receptor mRNA and endocannabinoid levels in the anterior pituitary gland. Biochem Biophys Res Commun (2000) 270:260-6. doi:10.1006/bbrc.2000.2406

49. Hill MN, Karacabeyli ES, Gorzalka BB. Estrogen recruits the endocannabinoid system to modulate emotionality. Psychoneuroendocrinology (2007) 32:350-7. doi:10.1016/j.psyneuen.2007.02.003

50. López HH. Cannabinoid-hormone interactions in the regulation of motivational processes. Horm Behav (2010) 58:100-10. doi:10.1016/j.yhbeh. 2009.10.005
51. Juraska JM, Sisk CL, DonCarlos LL. Sexual differentiation of the adolescent rodent brain: hormonal influences and developmental mechanisms. Horm Behav (2013) 64:203-10. doi:10.1016/j.yhbeh.2013.05.010

Conflict of Interest Statement: The authors declare that the research was conducted in the absence of any commercial or financial relationships that could be construed as a potential conflict of interest.

Copyright (c) 2015 Rubino and Parolaro. This is an open-access article distributed under the terms of the Creative Commons Attribution License (CC BY). The use, distribution or reproduction in other forums is permitted, provided the original author(s) or licensor are credited and that the original publication in this journal is cited, in accordance with accepted academic practice. No use, distribution or reproduction is permitted which does not comply with these terms. 